\title{
Advanced Segmented Silicon Space Telescope (ASSiST)
}

Richard G. Dekany, Douglas G. MacMartin, Gary A. Chanan, Mitchell Troy

Richard G. Dekany, Douglas G. MacMartin, Gary A. Chanan, Mitchell Troy, "Advanced Segmented Silicon Space Telescope (ASSiST)," Proc. SPIE 4849, Highly Innovative Space Telescope Concepts, (18 December 2002); doi: $10.1117 / 12.460563$

Event: Astronomical Telescopes and Instrumentation, 2002, Waikoloa, Hawai'i, United States 


\title{
Advanced Segmented Silicon Space Telescope (ASSiST)
}

\author{
Richard Dekany ${ }^{1}$, Douglas MacMartin², Gary Chanan ${ }^{3}$, Mitchell Troy ${ }^{4}$ \\ ${ }^{1}$ Caltech Optical Observatories, California Institute of Technology, \\ Pasadena, CA 91125 \\ ${ }^{2}$ Control and Dynamical Systems, California Institute of Technology, \\ Pasadena, CA 91125 \\ ${ }^{3}$ Physics and Astronomy, University of California, Irvine \\ Irvine, CA 94550 \\ ${ }^{4}$ Jet Propulsion Laboratory, California Institute of Technology, \\ Pasadena, CA 91109
}

\begin{abstract}
We propose thin silicon wafers as the building blocks of highly segmented space telescope primary mirrors. Using embedded MEMS actuators operating at high bandwidth control, this technology can achieve diffraction-limited image quality in the 3-300 micron wavelength range. The use of silicon wafers as cryogenic mirror segments is carried forward considering a point design of a future FAIR-class NASA ORIGINS mission.

We recognize four major economic factors that justify a massive paradigm shift in the fabrication of ultralightweight space telescopes:

- The precise process control and repeatability of silicon wafer manufacturing dramatically reduces the huge labor investment in mirror figuring experienced with Hubble Space Telescope.

- Once developed, the incremental cost of additional space telescopes based upon proven silicon manufacturing techniques can be very small. We estimate the marginal cost of a $30 \mathrm{~m}$ mirror when deploying a constellation can be as low as $\$ 36$ million (Year 2002 dollars).

- Federal R\&D funding in the area of microelectromechanical devices and advanced 3-D silicon processing is certain to have far greater economic return than similar investments in other technologies, such as optical membrane technology.

- The \$300B per year silicon processing industry will continue to drive increased MEMS functionality, higher product yields, and lower cost. These advances will continue for decades.

The intention here is to present the case for the economic advantage of silicon as a highly functional optical substrate that can be fabricated using unparalleled industry experience with precision process control. We maintain that many architectures superior to the ASSiST concept presented here are possible, and hope that this effort prompts future thinking of the silicon wafer telescope paradigm.
\end{abstract}

Keywords: Extremely large telescopes, space telescopes, silicon optics, segmented telescopes, MEMS actuators.

\section{INTRODUCTION}

Modern silicon processing techniques allow the fabrication of complex structural and dynamic elements out of silicon and its associated semiconductor materials. The rapid rise of microelectromechanical systems (MEMS) technology enables an entirely new paradigm for optical element fabrication. In particular, the development of MEMS 
actuators and sensors enables large segmented telescope mirrors of exceptionally low areal density. By integrating most of the control functions of these large structures into the optical segment itself, substantial mass reduction can be achieved.

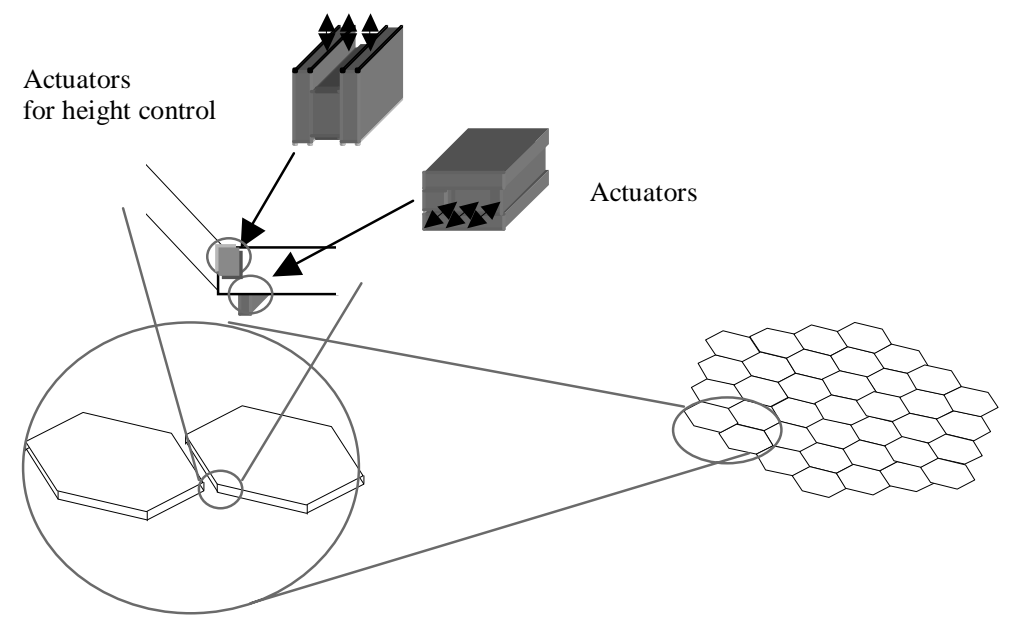

Figure 1. The role of MEMS technology in extremely large, highly-segmented mirror control. In this example two actuators are shown, one controlling relative segment height (piston) and one controlling the relative dihedral angle between segments (stacking). Two linear actuators along each segment edge can similarly control all necessary degrees of freedom.

The conceptual employment of MEMS actuators in the phasing of a segmented optical system is shown in Figure 1. MEMS actuators offer precise, high bandwidth control at lower mass and cost than conventional actuators. Moreover, the overdetermination of the control geometry allows active segment control on scales smaller than the segment diameter. The force that can be exerted by a MEMS actuator is limited but this is not a critical issue for a space-based telescope in a zero gravity environment.

Our experience with precision optomechanics has shown that 1) precision structures require precision tooling and 2) cost-effectiveness requires precision process control. The former statement is simply a recognition that optical structures require optical or similarly precise techniques of construction and testing. The latter statement is a recognition of the high cost of human labor and the economic advantage of automation.

These principles have been employed in the fabrication of capital-intensive equipment such as within the aircraft and microelectronics industries, with spectacular success. With a vision of the cost-effective precision development of proposed third-generation NASA Office of Space Science (OSS) ORIGINS missions, such as Life Finder (LF) and Planet Imager (PI), we maintain that these same principles can and ought to be applied to large gossamer apertures for use in space.

The goal of ASSiST is to establish a new paradigm for the development of large space optics, replacing the heavy, costly, 'one-off' nature of past designs with a precision controlled, automated, repeatable process for lightweight mirror segment fabrication.

\section{SCIENCE GOALS}

The NASA ORIGINS Program defining science questions, "Where do we come from?" and "Are we alone?" engage both scientific and public minds. Each of the highest-level science goals,

- To understand how galaxies formed in the early universe

- To understand how stars and planetary systems form and evolve

- To determine whether habitable or life-bearing planets exist around nearby stars

- To understand how life forms and evolves 
share a common requirement for their answer: the development of extremely large optical and infrared collecting apertures for use in space. Due to foreseeable limitations on available launch capability, current optics manufacturing techniques are incapable of meeting ORIGINS requirements. ASSiST provides a new architecture for telescope construction that meets ORIGINS requirements.

Although the ORIGINS program covers a broad wavelength range, the impact of ASSiST will be particularly relevant to science in the mid-infrared $(3-30 \mathrm{~m})$, where increasing sensitivity can be exploited to address unique science questions, and in the far-infrared $(30-300 \mathrm{~m})$ a regime that remains deficiently explored. The proposed development will help fill the large scientific void between the capabilities of Next Generation Space Telescope (NGST) and the capability of the future Atacama Large Millimeter Array (ALMA). By increasing resolution as well as sensitivity, the very large aperture telescopes enabled by this effort will additional overcome the confusion limit imposed upon smaller aperture space telescopes.

Within the range of $3 \mathrm{~m}$ to $300 \mathrm{~m}$, large apertures are particularly well suited to addressing the following key ORIGINS science programs:

- The resolution of the far infrared high redshift background into individual galaxies and protogalaxies, separating overlapping halos and refining the early star formation rates

- Birth and evolution of stars and planetary systems in the nearest star-forming regions. Spectroscopy of cold cloud collapse would reveal the dynamical mechanisms of star formation as well as disk and outflow dynamics for stellar ages $<10,000 \mathrm{yrs}$

- Molecular hydrogen and C+ emission of gas clouds in the early Universe, tracing gas dynamics in the era prior to metal formation

- Giant planet formation in debris disks, testing formation and planet migration theories

- Thermal emission spectroscopy of Kuiper Belt Objects, revealing their surface chemistries.

\section{ASSiST MISSION CONCEPT}

To provide a framework to discuss the advantages and challenges of silicon wafer telescopy, we adopt a baseline set of mission requirements that meet ORIGINS science goals in one particular wavelength regime, the mid-to far-infrared wavelength range. Note that this particular selection does not limit that potential application of silicon segment technology in other architectures.

Operating wavelength range:

Entrance pupil diameter:

Unvignetted field of view:

Structure operating temperature:

Aperture Areal Density:

Other:

\section{3 - 300 microns \\ 30 meters \\ 6 arcmin diameter}

$30-50 \mathrm{~K}$

$3 \mathrm{~kg} / \mathrm{m} 2$

Low thermal emissivity, low consumption of expendables, efficient packaging, survival during launch, on-orbit deployment, and redundancy in critical subsystems

The baseline optical design is a modified Schmidt telescope, composed of an aspheric (but zero power) reflecting corrector mirror, followed by an F/10 hyperbolic primary mirror. Each mirror is highly segmented in nature, approximately 30 meters in diameter, and thermally and dynamically isolated behind a large sunshield. The basic configuration for ASSiST is presented in Figure 2.

The modified-Schmidt corrector mirror also serves as the pointing mirror, selecting the scientific target of the telescope from a 5 degree field of regard. Operationally, observations would be made within a single field of regard for extended periods, dramatically reducing both the need to repoint the constellation and the torques transmitted to the mirror surfaces.

The telescope alignment is maintained through formation flying of the two mirrors. Optical tolerancing of this configuration results in a position keeping requirement of $\sim 1 \mathrm{~cm}$ in the transverse directions and a requirement for an active focus stage and fine-pointing mirror feeding the instrument package.

Importantly, we take advantage of ORIGINS technologies assumed to be demonstrated during the coming one to two decades, namely precision formation flying, cryogenic operation and instrumentation, and precision structural 
deployment that will be necessarily demonstrated for Next Generation Space Telescope (NGST) and Terrestrial Planet Finder (TPF). Thus, we concentrate on the unique technologies and issues for ASSiST in our remaining discussions.

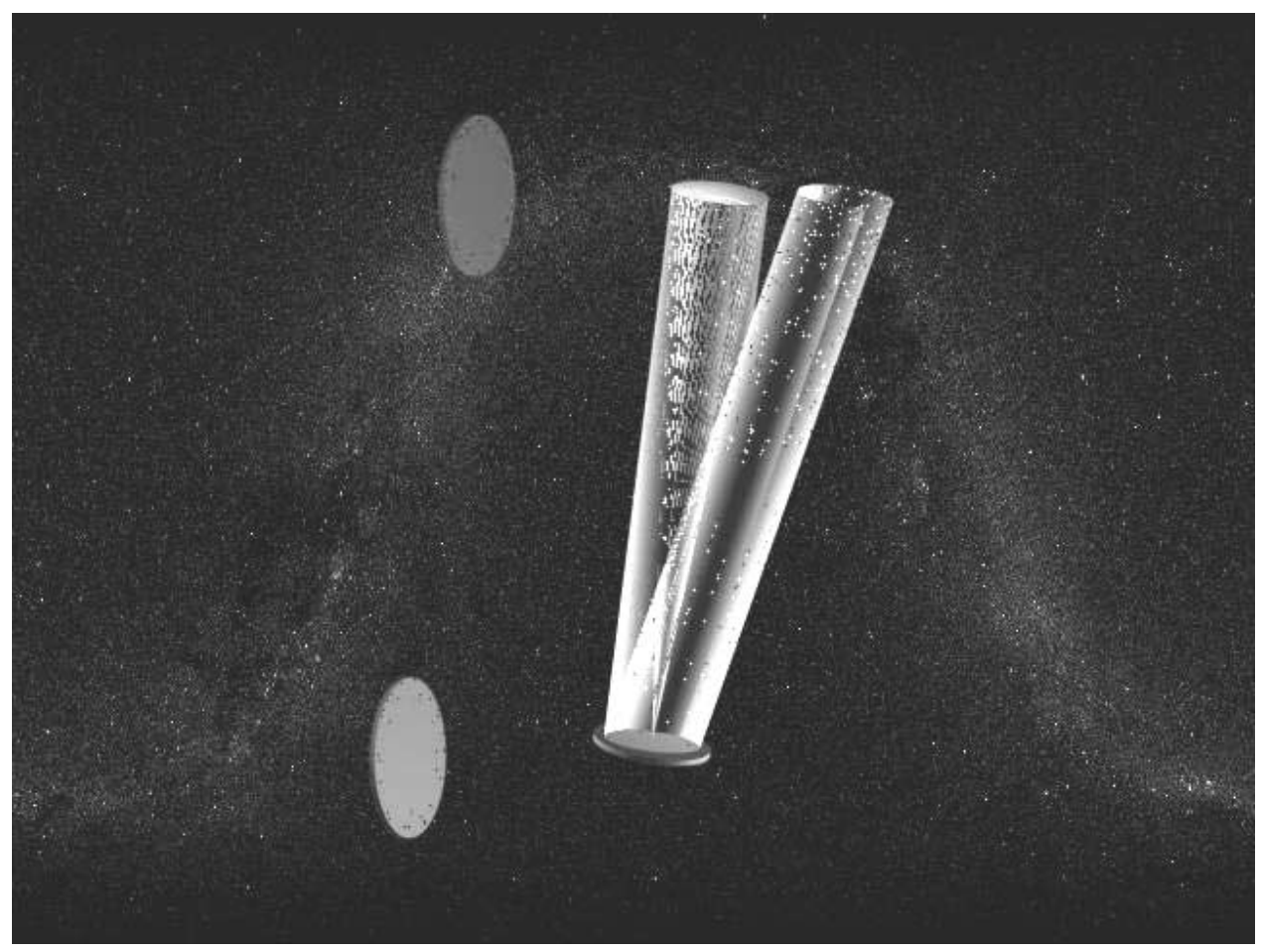

Figure 2. Spacecraft formation flying geometry for ASSiST. Starlight entering from the top-right is collected by a Schmidt corrector mirror (bottom) then focused by the F/10 primary mirror (top). Also shown are two large sunshades (at left), which may be inflatable. The instrument bus resides behind the corrector mirror.

\section{PRIMARY MIRROR ARCHITECTURE}

Although highly segmented primary mirrors have been previously considered, the control challenges have in general been deemed too great to guarantee the feasibility of the concept. We address these concerns in two ways. First, unlike previous highly segmented concepts of up to $10^{5}$ segments, the ASSiST primary has only 12,000 mirror segments. For comparison, it should be noted that the current planning for large ground based telescopes includes concepts for a 1,080 segment, $30 \mathrm{~m}$ diameter telescope ${ }^{1,2,3}$, and an $\sim 2,000$ segment $100 \mathrm{~m}$ diameter telescope ${ }^{4}$. Moreover, the ASSiST primary mirror architecture consists of a hierarchical distribution of $300 \mathrm{~mm}$ diameter segments assembled into $3 \mathrm{~m}$ diameter rafts, which are in turn assembled into a $30 \mathrm{~m}$ diameter primary mirror. The MEMS control occurs only on the raft level (between individual segments), while the rafts themselves are assumed to be controlled using mesoscale actuators. For now, we choose to concentrate on the controls problem within a raft, which is a problem with only a few hundred degrees of freedom, and well within the controls experience of ground-based segmented telescopes.

A unique feature of the ASSiST concept is the absence of a stiff backplane against which individual segments may react. Rather, we envision forces between segments transmitted exclusively between segments, along segment edges. Figures 3 and 4 depict the situation.

Wavefront control

Our concept for the geometry of the primary mirror control is shown in Figure 5, where we indicate two linear (piston) actuators along each segment boundary, with a raft. For 2 actuators along each segment edge, there are 
(ignoring perimeter effects) $6 \mathrm{~N}$ degrees of freedom that must satisfy $3 \mathrm{~N}-3$ rigid body constraints and $3 \mathrm{~N}$ flexural constraint. Each segment can be moved in tip, tilt, and piston relative to its neighbors, as well as twisted to change the value of it's $\alpha_{22}$ (astigmatism) coefficient, if necessary (edge actuators are unable to affect the $\alpha_{20}$ (focus) coefficient). Details of the control law for this architecture are currently under development.

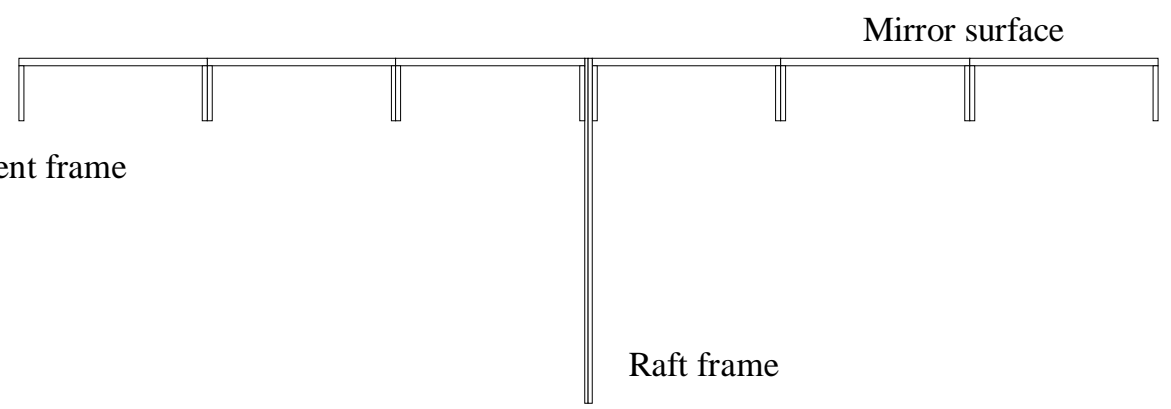

Figure 3. The cross-sectional architecture of the ASSiST primary mirror. Individual segments consist of $1 \mathrm{~mm}$ thick, $300 \mathrm{~mm}$ diameter silicon wafers with $\sim 10 \mathrm{~mm}$ deep frames, assembled into $3 \mathrm{~m}$ diameter rafts having $100 \mathrm{~mm}$ deep frames, This view shows 6 segments on edge, on either side of a raft boundary. For clarity, the vertical dimension of this figure is expanded roughly 10x relative to the horizontal dimension (i.e. individual segments have width to depth aspect of $~ 30: 1$ ).
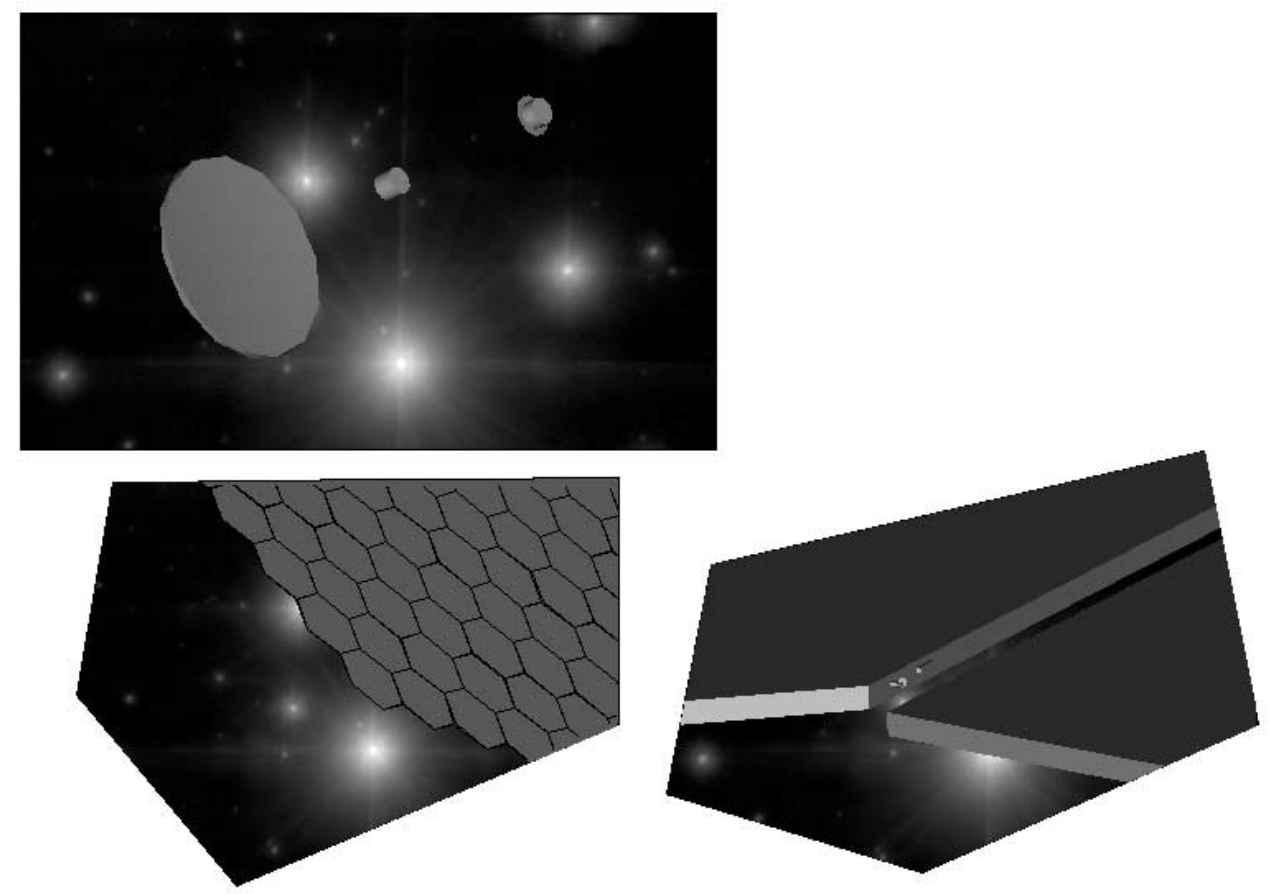

Figure 4. Three views zooming in on the location of segment actuators. Located on the outside faces of $10 \mathrm{~mm}$ deep segment frames, this architecture uses no heavy backplane against which to push. The third panel shows two segments artificially separated in order to view the actuators. In practice, the segment gaps width is $\sim 30$ microns. This exquisite control makes diffraction from the segment gaps negligible, even for observations down to a faintness of $m_{L}=30$. 


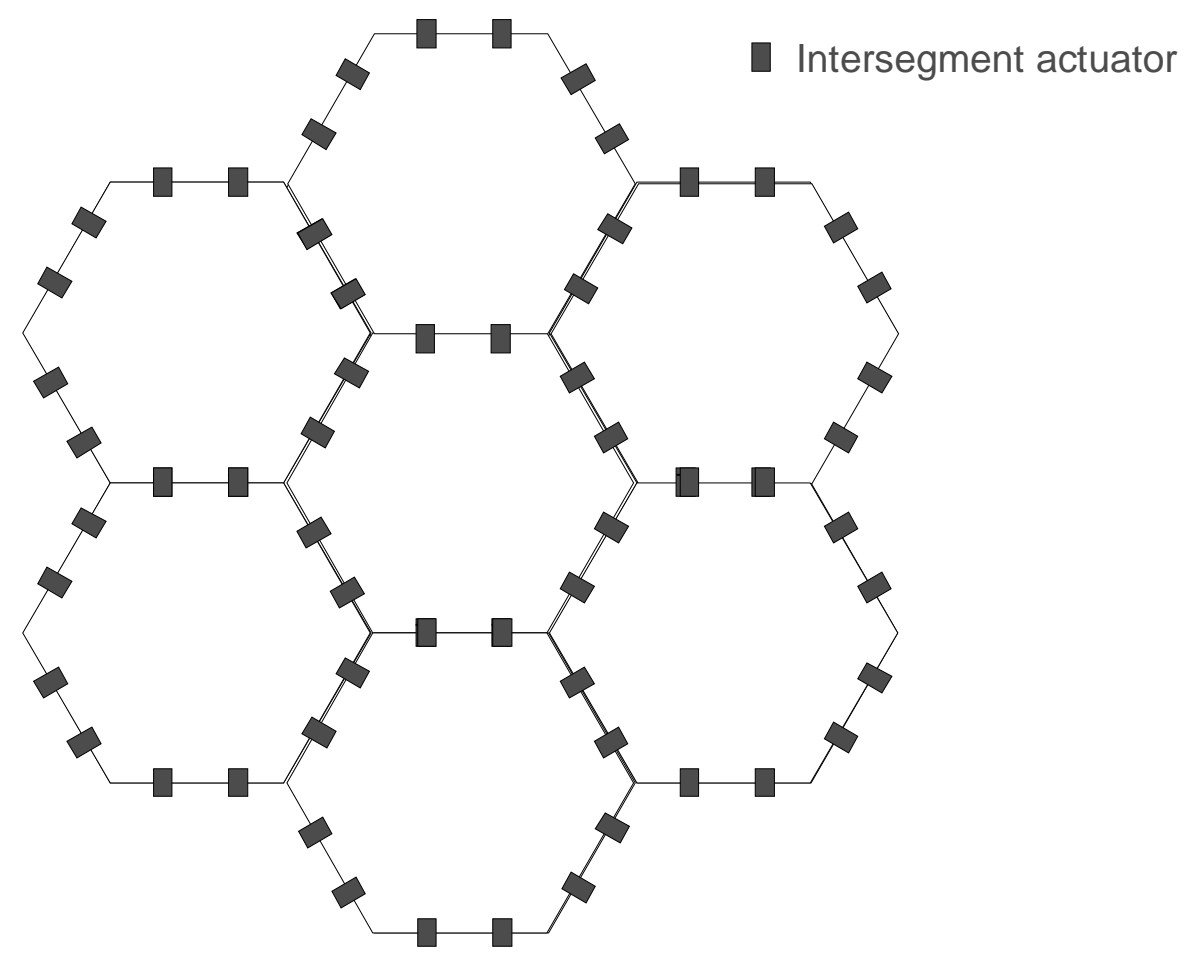

Figure 5. Top view of a portion of a single ASSiST raft, showing 7 segments and the location of intersegment actuators. The overdetermination of the geometry allows for both redundancy in the active control of the rigidbody degrees of freedom, and the potential for low-order, non-rigid body control of individual segment figure.

Establishment and maintenance of an optically precise measuring system is crucial to the scientific success of ASSiST. As an initial approach, proven successfully at the Keck Telescopes, we can use a large format science camera, in a defocused state, to provide stacking information for all the segments in a raft. Phasing within a raft can be accomplished through either a dedicated wavefront sensor camera or with the phase retrieval techniques developed by D. Redding for $\mathrm{NGST}^{5}$. After individual rafts are internally phased, these would be aligned and phased relative to each other using the same technique. High-bandwidth segment-to-segment wavefront sensing can be implemented using either local one-to-one sensor-to-actuator geometry (segment position keeping), or through a hierarchical implementation of relative segment piston information throughout the mirror. In all cases, a high-order $(10,000$ actuator), high-bandwidth $(\sim 500 \mathrm{~Hz},-3 \mathrm{~dB})$, large stroke (10-20 microns) adaptive optics system utilizing natural guide stars brighter than $\mathrm{m}_{\mathrm{H}}=14$ is expected to be required to eliminate residual mirror and spacecraft vibrations ${ }^{6}$. Fast guiding of tip/tilt and focus errors will be sensed with an on-instrument fast wavefront sensor.

During maneuvers in flight, the amount of control effort needed to cope with the mechanical behavior of the mirror will depend on the specific slew accelerations and other disturbances. Strong resonances or traveling waves through the structure may occur. Dynamic behavior (resonance frequencies, response functions, etc) has not yet been studied in detail. It is reasonable to foresee that special attention must be paid to both the mechanical stiffness and the damping between all connected elements: mirror segments and rafts.

When turning to acquire a new astronomical target, the pointing mirror of the two-mirror ASSIST concept will experience centripetal accelerations that potentially could tear segments away from their immediate neighbors. We have estimated that centripetal accelerations of $4 \times 10^{-9}\left[\mathrm{rad} \mathrm{s}^{-2}\right]$ provides a factor of 100 safety margin over the holding strength possible with simple piezoelectric actuators. This restricts ASSiST repointing to slow rates, on the order of a degree per minute.

ASSiST will not attempt to maintain it's surface figure during repointing, but it is expected to settle to within acceptable vibration levels within several minutes, through active damping via the segment actuators. Careful minimization of disturbances during operations is a major design driver for future spacecraft development. 
Segment Microgaps

To avoid thermal background radiation and minimize scattered radiation, the gap between the mirror segments must be less than a few tens of micrometers. This implies that both pre-flight (for assembly within a raft) and in-flight (for assembly of rafts) one might like to have a coarse attachment, followed by an accurate adjustment of the distances down to the required $200 \mathrm{~nm}$ level of accuracy.

For hexagonal segments, the ratio of gap area to segment area goes as the gap width divided by segment edge length. The average contamination flux density in the focal plane goes as the gap area divided by the gap width squared (assuming here a uniform flux instead of a diffraction pattern). Thus the effect of light scattering on science observations, in terms of scattered halo intensity, goes roughly as the cube of the gap width. Standard wafer dicing operations allow a gap width of only $30 \mu \mathrm{m}$, which gives exceptionally low scatter from a segmented telescope.

\section{ADVANTAGES OF SILICON AS AN OPTICAL SUBSTRATE}

The use of silicon as an optical substrate material for ORIGINS missions has several key advantages ${ }^{7}$ :

- Superior thermal properties at cryogenic temperatures

- Quick and inexpensive superpolishing (<100 nm P-V; surface roughness $<0.5 \mathrm{~nm} \mathrm{rms}$ ). Note this drastically improves IR sensitivity by reducing the level of contamination caused by thermal emission of the sunshields scattering into the science path.

- Lightweight, even compared to beryllium, silicon carbide, or graphite epoxy.

Beyond its material properties, silicon can reduce the mass of gossamer aperture by allowing the designer to embed typical spacecraft functions directly into the optical segment itself. Thus, instead of the traditional optical substrate, which does nothing except reflect light in its first few angstroms of thickness, silicon segments additionally provide:

- Integrated segment edge sensors and MEMS actuators

- Embedded communications and power distribution networks

- On-segment logic and distributed processor capability

\begin{tabular}{|c|c|c|}
\hline Material Property & Value & Comment \\
\hline Density & $2.3 \mathrm{~g} / \mathrm{cm}^{3}$ & $\begin{array}{c}\text { Less than } \mathrm{SiC}, \text { about } 50 \% \text { greater than } \\
\text { polyimide membrane }\end{array}$ \\
\hline Young's modulus & $1.9 \times 10^{12} \mathrm{dyne} / \mathrm{cm}^{2}$ & \\
\hline Yield strength & $7.0 \times 10^{10}$ dyne $/ \mathrm{cm}^{2}$ & \\
\hline Thermal conductivity & $157 \mathrm{~W} / \mathrm{m} \mathrm{K} @ 300 \mathrm{~K}$ & Better than SiO, not as good as SiC \\
\hline Thermal conductivity & $5130 \mathrm{~W} / \mathrm{m} \mathrm{K} @ 35 \mathrm{~K}$ & Better than SiC, and most other materials \\
\hline Thermal expansion coefficient & $2.33 \times 10^{-6} / \mathrm{K} @ 300 \mathrm{~K}$ & Comparable to SiC \\
\hline Thermal expansion coefficient & $0.5 \times 10^{-6} / \mathrm{K} @ 35 \mathrm{~K}$ & Comparable to Zerodur \\
\hline Coatings & Yes & $\mathrm{Ag}, \mathrm{Au}$, other infrared coatings possible \\
\hline
\end{tabular}

Table 1. Physical properties of silicon ${ }^{8,9}$.

Economically, silicon has powerful advantages:

- Optical quality segments can be made inexpensively. Currently (2002), polished $300 \mathrm{~mm}$ wafers can be obtained for $\sim \$ 100$. Thus the material cost of the primary mirror silicon is only $12,000 * \$ 100=\$ 1.2 \mathrm{M}$. With additional figuring, testing, MEMS functionality, and special handing requirement to avoid breakage, we estimate individual segments costs can be made as low as $\$ 3,000$ in volume. Thus, the replication cost when 
launching an array of $30 \mathrm{~m}$ telescopes (for missions such as NASA's Life Finder) may only be $\sim \$ 36 \mathrm{M}$ per telescope (not including launch costs, and after substantial technology development).

- Redundancy, in both actuation and other functionality, can be incorporated with huge cost savings. Once the fabrication process is in place, there is very small incremental cost of redundant systems.

- Federal R\&D funding in the area of microelectromechanical devices and advanced 3-D silicon processing is certain to have far greater economic return than similar investments in other technologies, such as optical membrane technology.

- The \$300B per year silicon processing industry will continue to drive increased MEMS functionality, higher product yields, and lower cost. These advances will continue for decades.

It is important to note that although the development of all the necessary segmented silicon mirror technology may be expensive, once this investment is made the incremental cost of additional 30 meter mirrors can be made very small. This provides a key motivation for the investigation of silicon segments, namely that the technology is cost-effectively scalable to large constellations of interferometrically combined apertures necessary to achieve the ultimate ORIGINS goals of high resolution imaging of Earth-like planets around nearby stars.

To meet the areal density goal of $3 \mathrm{~kg} / \mathrm{m}^{2}$, we calculate that the thickness of individual silicon segments must be approximately $1 \mathrm{~mm}$. Fortunately, standard silicon processing techniques are capable of polishing very flat wafers of this thickness (this is driven by the stringent depth-of-focus requirement of high numerical aperture photolithography).

Individual segments of the primary mirror must have a radius of curvature of 600 meters, corresponding to $17.5 \mu \mathrm{m}$ of spherical sag relative to a flat surface. Although a manufacturing complication, the feasibility of stressed segment polishing has already been demonstrated for silicon wafers, as has figuring of optical power using etching techniques wherein diffused dopants control the etch rate of the wafer surface, providing optical power. Alternatively, the full $17.5 \mu \mathrm{m}$ curvature could be realized completely by means of a set of segment actuators. Note that the segments that constitute the corrector mirror in the modified-Schmidt concept for ASSiST can be nominally without optical power.

\section{MEMS Integration}

ASSiST relies on very precise, internally stiff, high bandwidth actuators using microelectromechanical (MEMS) processing techniques. While these actuators may be used in the classical sense to push against some form of backplane, the high temporal bandwidth of MEMS technology makes possible the interesting option of phasing a mirror without the need of a heavy backstructure. Rather, segment tip/tilt would be maintained through forces conducted within the plane of individual segments, while segment piston, which is purely a relative motion, would be maintained through forces normal to the segments. Thus the figure control actuators provide a unique adaptive structural linkage between the many individual segments. Because of the small-scale sizes of MEMS actuators, typical tens of microns, they are, importantly, capable of operation up to kilohertz bandwidths, providing high effective stiffness from control. Although this same scale size limits the forces that MEMS actuators can exert, this limitation is not severe when positioning very thin (and thus low moment of inertia) segments in space.

Typical MEMS devices are actuated by thermopneumatic, bi-metallic, shape memory alloy, electrostatic or piezoelectric mechanisms. Considering force, displacement (or deflection), consumed power, actuation time and fabrication feasibility based on the requirements of segmented mirrors, we have derived a preliminary MEMS actuation concept, using inchworm piezoelectric actuators to control height and angle between segments. Although piezo ceramic loses about $80 \%$ of its strain at $30 \mathrm{~K}$, an inchworm with extra material in the clamps and extension section can provide the sufficient strain. Further investigation of cryogenic material options, such as strontium titanate, should also be considered.

Usually, MEMS actuators cannot be fabricated directly on a sidewall of silicon wafer; so we propose using the LIGA process, an X-ray lithography technique in which a polymer film is exposed to x-ray radiation, changing the composition and allowing three dimensional removal of material. Advantages of LIGA technology include:

- Broad range of materials: metals, alloys, plastics, ceramics (e.g. piezo ceramics)

- Variety of designs possible

- Structures over $1 \mathrm{~mm}$ high

- High precision into the sub $\mu \mathrm{m}$ range

- Low cost mass production 
One of the key drivers for segment and actuator concepts for ASSiST is the segment configuration constituting the primary and corrector mirrors. Depending on segment geometry, assuming $300 \mathrm{~mm}$ diameter wafer technology, ASSiST requires a total number of segments between 10,000 and 15,000. Although this is a large number, we note that active mirrors for ground-based adaptive optics have been constructed with over a thousand segments and that digital MEMS projector system, under development at Texas Instruments and elsewhere, consist of 1040 x 780 micromirror arrays. We note that $300 \mathrm{~mm}$ wafer technology is the current state of the art, but is expected to become the industry standard in the coming 2-3 years, allowing this technology to fully mature on the time scale required for ASSiST.

\section{OUTSTANDING ISSUES}

The point concept for an ASSiST mission does not begin to address a host of difficult technological issues, among the foremost of which is ground-based testing of large, gossamer aperture, survival of mirror segments and rafts during launch vibrational and acoustic loading, raft deployment and self-assembly, and cryogenic thermal control. It is out belief, however, that unlike highly segmented space telescope concepts of the past, the maturation of MEMS technology bolsters the feasibility of silicon as an infrared and far-infrared mirror material that deserves future investigation.

\section{REFERENCES}

\footnotetext{
${ }^{1}$ Nelson, J., E., "Design concepts for the California Extremely Large Telescope (CELT)", Proc. SPIE 4004, 282-289, (2000).

${ }^{2}$ Dekany, R., et al., "Design considerations for CELT adaptive optics", Proc. SPIE 4004, 212-225 (2000).

${ }^{3}$ Dekany, R., et al., "Initial concepts for CELT adaptive optics", Proc. SPIE 4839, (2002).

${ }^{4}$ Dierickx and Gilmozzi, "Progress of the OWL 100-m telescope conceptual design," Proc. SPIE 4004, 290-299, (2000).

${ }^{5}$ Redding, D., et al., "Image-based wavefront sensing and control experiments," Proc. SPIE 4850 (2002).

${ }^{6}$ Within the relatively large 6 arcmin diameter FoV of ASSiST, such stars are likely to exist with high probability, even near the galactic poles. This is a large motivation for the wide-field modified Schmidt telescope point design we present here.

${ }^{7}$ These advantages have been previously identified by Jacoby, et al., Proc. SPIE 4451, 67-76, (2001) and elsewhere.

${ }^{8}$ Wolf, S. and Tauber, R.N., Silicon Processing for the VLSI Era, Volume 1 - Process Technology, Lattice Press, Sunset Beach, California (1986).

${ }^{9}$ Helvajian, H., Microengineering Aerospace Systems, Aerospace Press, El Segundo, California (1999).
} 